\title{
Ueber das Wachs der Hummeln (Bombus sp.).
}

\author{
Von
}

Ernst Erw. Sundwik.

(Der Redaction zugegangen am 8. August 1898.)

Das gewöhnliche Bienenwachs ist allbekannt. So viel ich aber auch nachgesucht habe, habe ich keine chemischen Untersuchungen über das Wachs der Hummeln, dieser wichtigen Abtheilung der grossen Bienenfamilie, gefunden. Spärliche Notizen über die Absonderung und Verwendung desselben findet man in entomologischen Werken, besonders in Spezialwerken über die Hummeln.

Die Quelle, aus welcher ich meine Kenntniss vom Hummelwachs hauptsächlich geschöpft habe, ist die schöne Monographie von Hoffer, "Die Hummeln Steiermark's, Lebensgeschichte und Beschreibung derselben», Graz 1882. Hoffer sagt, dass das Hummelwachs nicht so rein, weich und schön ist, wie das der Honigbiene. Es hat eine schmutzigweisse Farbe, ist sehr brüchig und lässt sich nur schwer zwischen den Fingern kneten. Er spricht hier vom Wachse, welches er von den Báuchsegmenten vorsichtig abgestreift hatte. Ich bemerke hier gleich, dass ich solches reines Wachs nicht habe untersuchen können. Dagegen habe ich Wachs zur Untersuchung gesammelt, welches von den Hummeln $\mathrm{zu}$ verschiedenen Zwecken in den Nestern aufgespeichert wird.

Mit Pollen zusammengeknetetes Wachs findet man als seichte Hügel oder Leistchen, in welche die Eier bezw. die Larven eingeschlossen sind. Ebenso findet man mit Wachs nur wenig vermengte Pollen in grösseren Ansammlungen als Futter für die ausgekrochenen jungen Hummeln bestimmt. Ich habe durch Abstreifen dieser Wachspollensammlungen und Befreien derselben von Eiern und Larven nicht unbeträchtliche Mengen Rohstoff erhalten können. Durch Befreien der Cocons von Larven und Auskochen mit geeigneten Lösungsmitteln habe ich das auf der Aussenseite der Cocons haftende Wachs ausgezogen. Dieses war jedoch ziemlich stark mit Fett gemengt. 
Ich fing schon 1892 mit den Untersuchungen an, bekam aber erst 1896 etwa 35, 1897 mehr als 60 Nester zur Untersuchung. Fast alle stammten von Bombus muscarum, nur einige wenige von Bombus lapidarius. Da sich aber in dem Wachse beider Arten dieselben chemischen Verhältnisse zeigten, habe ich später stets die Rohprodukte aus verschiedenen Nestern gemischt bearbeitet. Aus 130 gr. Wachspollenmasse bekam ich durch Ausziehen mit Aether oder Chloroform etwa 30 gr. rohes Wachs von braungelber Farbe (Schmelzpunkt $35-40^{\circ}$ C.). Der Geruch war sehr angenehm, honigartig, gar nicht ranzig. Die Farbstoffe, ebenso Honig und alle in Wasser löslichen Stoffe hatte ich vor der Extraction mit Aether durch längeres Einsenken des Wachses bezw. der Cocons in fliessendes Wasser möglichst entfernt.

Beim Liegen im Tageslicht wurde der dünne Wachskuchen fast vollständig gebleicht und weiss. Dagegen wurde der Geruch deutlich ranzig.

Hoffer sagt (a. a. O.: Seite 40), «dass die Hummeln vor dem Gebrauche das Wachs mit verschiedenen harzigen und anderen klebrigen Stoffen vermengen, so dass es in diesem Falle kaum als Wachs zu erkennen ist; schmelzbar, aber in geringerem Grade, bleibt es auch dann noch, und den charakteristischen Wachsgeruch zeigt es in grösseren Massen immer».

Ich glaube, dass diese Behauptungen nicht das Richtige treffen. Harzige Einmischungen würden wohl auf den Geruch ihre Wirkungen ausüben. Die dunkle, braune Farbe stammt vom Pollen, wenn auch vielleicht nicht ausschliesslich. Ich glaube, dass das von mir extrahirte Rohwachs hauptsächlich nur von Fett und etwas Pollenfarbstoff verunreinigt war. Das mit vielleicht $70 \%$ Pollen vermengte Wachs kann selbstverständlich nur wenig oder kaum schmelzen, sondern nur erweichen.

Da dieses Wachs zu einem anderen Gebrauche als das Bienenwachs bestimmt ist, so ist es einleuchtend, dass die Zusammensetzung in chemischer Hinsicht eine ganz andere sein muss. Dieses hat auch die chemische Untersuchung erwiesen.

Das Reinigen des Rohwachses ist überaus schwer, da das beigemengte Fett fast dasselbe Lösungsverhältniss zeigt 
wie das Hauptprodukt. Ich hatte schon im Jahre 1896 gefunden, dass dieses kein Ester, sondern sehr wahrscheinlich ein Alkohol ist, und dass die, verunreinigenden Glycerinester durch Saponification leicht zu entfernen sind. Ich habe dann später in der Weise gearbeitet, dass ich das Rohwachs mit schwächerer Kalilauge auf dem Wasserbade behandelte, dann das oben abgeschiedene Wachs mehrmals aus Alkohol umkrystallisirte. Die Verluste sind dennoch gross. Der Stoff löst sich ziemlich leicht in kochendem Alkohol und scheidet sich um so vollständiger aus, je reiner er ist. Nach sechs- bis siebenmaligem Umkrystallisiren erhöht sich der Schmelzpunkt auf $69-70^{\circ} \mathrm{C}$. ; nach weiterem Umkrystallisiren bekommt man schliesslich ein Produkt, das bei $74-75^{\circ}$ C. schmilzt.

Der bei $69-70^{\circ}$ C. schmelzende Stoff (und ebenso die niedriger schmelzenden Produkte) besitzt gewöhnlich die klebrige Beschaffenheit des gemeinen Bienenwachses, der reine, bei $75^{\circ}$ C. schmelzende Stoff aber nicht mehr. Was Knetbarkeit betrifft, gleicht es völlig dem reinen, von Hoffer beschriebenen, von den Hummeln selbst abgeschabten Wachse.

Aus Alkohol krystallisirt bildet der Stoff ausserordentlich feine, biegsame, wollige Nadeln, die die ganze Mutterlauge zum Gestehen bringen. Durch Auswaschen mit Alkohol und Auspressen bekommt man einen schwach gelblichen Presskuchen, der bald durch Verwitterung weiss wird. In geschmolzenem Zustande zeigt das Wachs eine sehr schwache gelbliche Farbe, obgleich der rohe Wachskuchen vor dem Umkrystallisiren durch Liegen in der Sonne fast gänzlich gebleicht wird. Die nach dem Schmelzen wieder erstarrte, etwas trübe Masse ist krystallinisch, hart, haftet fest am Glase und wird nach dem Reiben beim Losmachen stark elektrisch, wodurch alle feineren Theile herumgeschleudert werden oder an anderen Gegenständen haften bleiben. - Der Stoff löst sich auch in der Kälte mehr oder weniger leicht in den gewöhnlichen Lösungsmitteln für Fette: Chloroform, Aether u. s. w.

Hier folgen zwei Analysen, die ich gemacht habe:

I. Schmelzpunkt der Substanz $73-73, \check{0}^{\circ} \mathrm{C}$.

0,32165 gr. gaben $0,9697 \mathrm{CO}_{2}=82,23 \% \mathrm{C}_{2}$ und $0,4082 \mathrm{H}_{2} \mathrm{O}=14,10 \% \mathrm{H}_{2}$. 
II. Die vorige Substanz noch mehrmals umkrystallisirt, wodurch der Schmelzpunkt auf $74,5^{\circ}$ C. erhöht wurde.

0,250 วั3 gr. gaben $0,7714 \mathrm{CO}_{2}=82,41 \% \mathrm{C}_{2}$ und $0,3286 \mathrm{H}_{2} \mathrm{O}=14,30 \% \mathrm{H}_{2}$.

Die erste Analyse stimmt leidlich, die zweite sehr gut mit der Formel $\mathrm{C}_{34} \mathrm{H}_{70} \mathrm{O}$, welche $82,59 \% \mathrm{C}$ und $14,17 \% \mathrm{H}$ erfordert. Doch muss es weiteren Untersuchungen überlassen werden, die endgiltige Formel festzustellen, da die gleich unten folgende Untersuchung nicht Resultate ergeben hat, welche die Formel $\mathrm{C}_{34} \mathrm{H}_{70} \mathrm{O}$ zweifellos machen.

Beim Erhitzen der Substanz bei $150-160^{\circ}$ C. mit Benzoesäureanhydrid $\left(\mathrm{C}_{54} \mathrm{H}_{70} \mathrm{O}\right.$ gegen $0,5 \mathrm{Mol}$. Benzoesäureanhydrid) erhält man eine sowohl in heissem als, obwohl weniger leicht, in kaltem Alkohol lösliche Substanz, die nach mehrmaligem Umkrystallisiren bei $55^{\circ}$ C. schmilzt. Diesen Schmelzpunkt erhielt ich bei zwei verschiedenen Präparaten. Auch durch Umkrystallisiren aus Benzol, Petroläther, Alkohol wurde er nicht geändert. Die Substanz musste also ziemlich rein gewesen sein. Die Analyse ergab folgende Werthe;

III. 0,3999 gr. Substanz gaben $1,2038 \mathrm{CO}_{\mathrm{z}}=82,104 \% \mathrm{C}_{\mathrm{z}}$ und $0,4766 \mathrm{H}_{2} \mathrm{O}=13,24 \% \mathrm{H}_{2}$.

Die Formel $\mathrm{C}_{34} \mathrm{H}_{69} .0 . \quad \mathrm{C}_{7} \mathrm{H}_{5} \mathrm{O}$ würde $82,27 \% \quad \mathrm{C}$ und $12,37 \% \mathrm{H}$ erfordern. Die hohen Wasserstoffprocente kann ich bis auf Weiteres nicht befriedigend erklären, um so mehr als vier verschiedene, hier nicht angeführte Analysen dieselben Wasserstoffprocente ergaben.

Es war meine Absicht, erst später diese Mittheilung einzusenden. Einige Umstände, vor Allem das geringe Material, welches $\mathrm{zu}$ meiner Verfügung steht, und die Unwahrscheinlichkeit, in diesem Sommer eine grössere Menge Rohmaterial zu erhalten, haben es jedoch nöthig gemacht, schon jetzt vorläufig meine Untersuchungen zu publiciren.

Helsingfors, Physiologisch-chemisches Institut. 